\title{
Congenital Anomalies Among Newborns in Diyala Province in
} 2017

\author{
Samara Mahmood Abdulhadi (BSC) ${ }^{1}$, Nadhim Ghazal Noaman $(\mathrm{PhD})^{2}$ and Shefaa \\ Mansour Hemza (FICMS) ${ }^{3}$ \\ Abstract
}

Background: Congenital anomalies or birth defects are groups of disordered of prenatal origin which can be caused by single gene defect, chromosomal disordered, multifactorial inheritance, and environmental teratogens.

Objective: To detect the incidence and types of congenital anomalies, as well as other associated factors.

Patients and Methods: This study was conducted in Albatool teaching hospital in Baquba city in Diyala government as a cross sectional study of 130 neonates with congenital anomalies from the period of 1st January to 31th December 2017. Patients were categorized according to Age of mother of neonate with congenital anomaly, address, residency, consanguinity between mother and father, mother's history of Diabetes Mellitus, periconceptional folic acid use, and abortion, also the sex of neonate, gestational age, birth weight, blood group and the type of congenital anomaly.

Results: Males were significantly affected (60\%) with congenital anomalies than females (37.7\%). The percentage of congenital anomalies was more $(68.46 \%)$ among neonates from consanguineous marriage than neonates from nonconsanguineous marriage $(31.54 \%)$. Baqubah city was recorded high percentage of anomalies(42.3\%), followed by Al-khalis district (24.6\%), and then Mukdadia district (15.5\%). High percentage of anomalies was recorded among neonates with mothers who did not use folic acid during peri conceptional period (57.7\%), and among multiparity (70.76\%), premature neonates $(57 \%)$, caesarean section $(60 \%)$, low birth weight neonates $(53.84 \%)$, neonates with blood group O (42.3\%), mother`s age 20-35 years (61.54\%), and rural residency(69.24\%), nervous system anomalies were the most common anomalies(43\%), followed by anomalies of circulatory system(21.7\%), and then chromosomal abnormalities(7.6\%).

Conclusion: The most common type of congenital malformations in Diyala government were nervous system malformations. Congenital malformations in Diyala Province were more common in Baquba city and AL Khalis district. Males were significantly affected more than females. Congenital anomalies were significantly more in offspring of consanguineous marriage than of non- consanguineous one. Folic acid usage during peri conceptional period was very important to reduce the incidence of congenital anomalies especially neural tube defects.

Keywords: Congenital anomalies, Newborn, Diyala. 
Corresponding Author: sh.man75@gmail.com

Received: $28^{\text {th }}$ August 2019

Accepted: $1^{\text {st }}$ September 2019

DOI:https://doi.org/10.26505/DJM.18024910828

\section{${ }^{1,3}$ AlBatool Teaching Hospital- Diyala-Iraq \\ ${ }^{2}$ College of Medicine - University of Diyala - Diyala -Iraq}

\section{Introduction}

Congenital anomalies are also known as birth defects, congenital disorders or congenital malformations. Congenital anomalies can be defined as structural or functional anomalies (for example, metabolic disorders) that occur during intrauterine life and can be identified prenatally, at birth, or sometimes may only be detected later in infancy, such as hearing defects[1].

Birth defects are still the leading cause of perinatal mortality and childhood disability in developed countries[2]. On the basis of clinical criteria, major malformations are morphogenetic defects producing function impairment needing medical or surgical treatment; defects not producing function Impairment and not requiring medical assistance are minor malformations(birth prevalence $<4 \%$ )or phenotypic variants (birth prevalence $>4 \%$ )[3].

Genes play an important role in many congenital anomalies. This might be through inherited genes that code for an anomaly, or resulting from sudden changes in genes known as mutations. Consanguinity (when parents are related by blood) also increases the prevalence of rare genetic congenital anomalies and nearly doubles the risk for neonatal and childhood death, intellectual disability and other anomalies. Some ethnic communities (such as Ashkenazi Jews or
Finns) have a comparatively high prevalence of rare genetic mutations such as Cystic Fibrosis and Haemophilia C[1]. A low socioeconomic status in a deprived neighborhood may include exposure to "environmental stressors and risk factors(4). Socioeconomic inequalities are commonly measured by the Cartairs-Morris score, Index of Multiple Deprivation, Townsend deprivation index, and the Jarman score. Environmental factors: are commonly known as teratogens. There are three main groups of teratogens: physical, chemical and biological[5].

Congenital malformations are important causes of infant death, both in absolute terms and as a proportion of all infant deaths, in both the developed and developing world. Although only a small percentage of all newborns, 2-3\%, are born with a major congenital malformation, congenital malformations account for nearly $20 \%$ of all infant deaths in developed countries. Based on World Health Organization data from 36 countries in different continents, overall infant mortality decreased on average $68.8 \%$ from 1950 to 1994 but infant mortality attributable to congenital anomalies decrease only $(33.4 \%)[3]$.

The history for a child with birth defects includes a number of elements that are 
related to etiologic factors. The first is the family history that is necessary to assess the inheritance pattern. It includes the pregnancy history of the mother (useful for recognition of recurrent miscarriages that may be a sign of a familial chromosomal disorder). The physical examination is essential to the diagnosis of a dysmorphic syndrome. The essential element of the evaluation is objective assessment of the structure of the child. Imaging Studies:Imaging studies can be critical in the diagnosis of a dysmorphic disorder. When there are abnormal neurologic signs or symptoms, central nervous system imaging is indicated. Other studies, such as echocardiography and renal ultrasonography, can be useful to identify additional major or minor malformations[6]. The prevention aims to ensure that individuals are born free of birth defects by being conceived normally and not being damaged in the fetal life. This can be achieved with basic reproductive health approaches which include family welfare services, promoting healthy dietary habits and lifestyle, safe food and environment; detecting, treating and preventing maternal infections; control of such diseases as insulin-dependent diabetes mellitus and epilepsy; vaccination, avoiding use of certain drugs during pregnancy and prior to conception (in women planning for the pregnancy)[1]. early surgical intervention is important to ensure complete recovery from congenital anomalies without residual defects[7]. The study aims to detect the incidence and types of congenital anomalies, as well as other associated factors.

\section{Patients and Methods}

This cross sectional study was conducted in Albatool teaching hospital in Diyala Province for the period from 1st January 2017 to $31^{\text {th }}$ December 2017. This work was done in the labour room and the theatre room where all neonates with congenital anomalies were examined, and collected, the information were taken from relatives of neonates and sometimes from mothers, some information were taken from. All cases were diagnosed by specialist pediatrician. Data were collected via a special design questionnaire, Patients were recognized according to: Age of mother of neonate with congenital anomaly, address, residency, consanguinity between mother and father, mother's history of Diabetes Mellitus, periconceptional folic acid use, and abortion, also the sex of neonate, gestational age, birth weight, blood group and the type of congenital anomaly.

\section{Statistical analysis}

Data analysis was done using SPSS 7.5 ,the results were presented in simple measures of frequency, Percentage, chisquare test to test the significance difference between proportions.P value of equal or less than 0.05 was considered as the level of significance.

\section{Results}

The total number of live birth in Albatool teaching hospital was $15907,7990(50.23 \%)$ were males and 7914(49.76\%) were females, sexual ambiguity recorded in $3(0.01 \%)$,from them 130 neonates with congenital anomalies 
$78(60 \%)$ were males and $49(37.7 \%)$ were significantly affected more than females, $\mathrm{p}$ females, which mean that males were value $=0.025$ as shown in Table (1).

Table (1):Distribution of congenital anomalies according to sex of neonate

\begin{tabular}{|c||c||c||}
\hline \multicolumn{1}{|c||}{ Sex } & $\begin{array}{c}\text { Total live birth } \\
\text { No. }(\boldsymbol{\%})\end{array}$ & $\begin{array}{c}\text { Congenital malformations } \\
\text { No. (\%) }\end{array}$ \\
\hline Male & $7990(50.23 \%)$ & $78(60 \%)$ \\
\hline \hline Female & $7914(49.76 \%)$ & $49(37.7 \%)$ \\
\hline \hline Sexual ambiguity & $3(0.01 \%)$ & $3(2.3 \%)$ \\
\hline Total & $15907(100 \%)$ & $130(100 \%)$ \\
\hline
\end{tabular}

$* \chi^{2}=370.4 ;$ d.f $=2 ; \mathrm{P}=0.0001$

$* \chi^{2}=5.01 ;$ d.f $=1 ; \mathrm{P}=0.025$

The percentage of congenital anomalies was high in Baqubah (42.3\%) followed by AL-khalis $(24.6 \%)$ followed by Muqdadia (15.5\%) followed by Baladroz (13.8\%), then followed by Khanaqeen (3.8\%).The most system involved was the nervous system, with the highest percentage in Baqubah (23.8\%) followed by both Al-khalis and Mukdadia (7.7\%),while Baladroz and Khanaqeen were recorded only $(2.3 \%)$ and

Table (2): Distribution of congenital malformations according to address of mothers of neonates with congenital malformations and system affected

\begin{tabular}{|c|c|c|c|c|c|c|}
\hline System affected & $\begin{array}{c}\text { Baqubah No. } \\
(\%)\end{array}$ & $\begin{array}{l}\text { Al-khalis } \\
\text { No. }(\%)\end{array}$ & $\begin{array}{l}\text { Mukdadia } \\
\text { No. }(\%)\end{array}$ & $\begin{array}{l}\text { Baladroz } \\
\text { No. }(\%)\end{array}$ & $\begin{array}{l}\text { Khanaqin } \\
\text { No. }(\%)\end{array}$ & $\begin{array}{l}\text { Total No. } \\
(\%)\end{array}$ \\
\hline Nervous system & $31(23.8 \%)$ & $10(7.7 \%)$ & $10(7.7 \%)$ & $3(2.3 \%)$ & $2(1.5 \%)$ & $56(43 \%)$ \\
\hline Circulatory system & $7(5.4 \%)$ & $10(7.7 \%)$ & $2(1.5 \%)$ & $8(6.15 \%)$ & $1(0.77 \%)$ & $28(21.7 \%)$ \\
\hline $\begin{array}{c}\text { Chromosomal } \\
\text { abnormalities }\end{array}$ & $8(6.1 \%)$ & $2(1.5 \%)$ & & & & $10(7.6 \%)$ \\
\hline $\begin{array}{l}\text { Genital organs } \\
\text { anomalies }\end{array}$ & $5(3.8 \%)$ & $1(0.77 \%)$ & & & $1(0.77 \%)$ & $7(5.4 \%)$ \\
\hline $\begin{array}{c}\text { Musculoskeletal } \\
\text { system }\end{array}$ & $1(0.77 \%)$ & $3(2.3 \%)$ & $3(2.3 \%)$ & & & $7(5.4 \%)$ \\
\hline Cleft/lip palate & $1(0.77 \%)$ & $3(2.3 \%)$ & $1(0.77 \%)$ & & & $5(3.8 \%)$ \\
\hline Digestive system & & $1(0.77 \%)$ & $10.77 \%)$ & $2(1.5 \%)$ & & $4(3 \%)$ \\
\hline Face(ear) & $1(0.77 \%)$ & & $1(0.77 \%)$ & & & $2(1.5 \%)$ \\
\hline U Urinary system & $1(0.77 \%)$ & & $1(0.77 \%)$ & & & $2(1.5 \%)$ \\
\hline Others & & $2(1.5 \%)$ & $1(0.77 \%)$ & $5(3.8 \%)$ & $1(0.77 \%)$ & $9(6.9 \%)$ \\
\hline Total & $55(42.3 \%)$ & $32(24.6 \%)$ & $20(15.5 \%)$ & $18(13.8 \%)$ & $5(3.8 \%)$ & $130(100 \%)$ \\
\hline
\end{tabular}

(1.5\%) respectively. Circulatory system was recorded major percentage in Al-khalis (7.6\%) followed by Baladroz $(6.15 \%)$ then Baqubah (5.4\%), chromosomal abnormalities were recorded major percentage in Baqubah $(6.1 \%)$ and $(1.5 \%)$ in Al-khalis, other systems were involved in small percentages in Diyala Province as shown in Table-2 and Figure(2) which represent descriptive Table. 


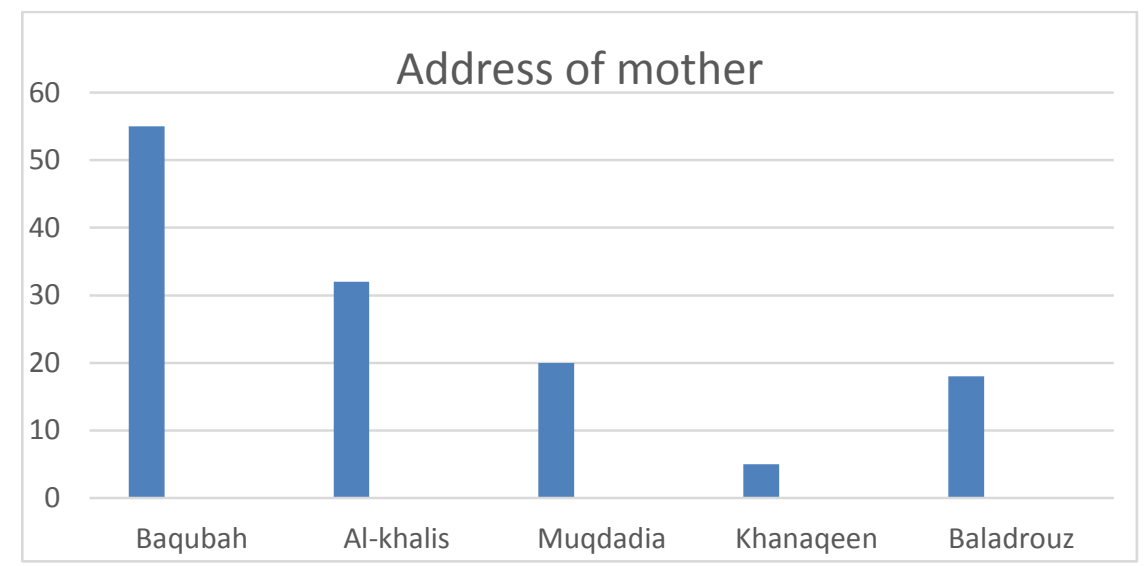

Figure(2): Distribution of congenital malformations according to Address of mothers of neonates with congenital malformations

Among 130 mothers studied for malformed neonate, the majority of them were multiparas 92(70.76\%), while 38(29.24\%) were primiparas. The most system involved in multiparas was the nervous system $43(33 \%)$, and $13(10 \%)$ in primiparas, followed by circulatory system $18(14 \%)$ in multiparas and $10(7.7 \%)$ in primiparas, followed by chromosomal abnormalities $8(6 \%)$ in multiparas and $2(1.53 \%)$ in primiparas then genital organs defects $5(4 \%)$ in multiparas and $2(1.5 \%)$ in primiparas, the musculoskeletal anomalies $4(3 \%)$ in multiparas and $3(2.3 \%)$ in primiparas, but this was statistically not significant $(\mathrm{p}=0.68)$ as shown in Table(3).

Table (3): Distribution of congenital malformations according to parity of mothers of neonates with congenital anomalies and system affected

\begin{tabular}{|l||c||c||c||}
\hline \multicolumn{1}{|c|}{ System affected } & $\begin{array}{c}\text { Primiparity No. } \\
(\%)\end{array}$ & $\begin{array}{c}\text { Multiparity No. } \\
(\%)\end{array}$ & Total No.(\%) \\
\hline \hline Nervous system & $13(10 \%)$ & $43(33 \%)$ & $56(43 \%)$ \\
\hline \hline Circulatory system & $10(7.7 \%)$ & $18(14 \%)$ & $28(21.5 \%)$ \\
\hline \hline Chromosomal abnormalities & $2(1.53 \%)$ & $8(6 \%)$ & $10(7.6 \%)$ \\
\hline \hline Genital organs anomalies & $2(1.53 \%)$ & $5(4 \%)$ & $7(5.4 \%)$ \\
\hline \hline Musculoskeletal system & $3(2.3 \%)$ & $4(3 \%)$ & $7(5.4 \%)$ \\
\hline Cleft/lip palate & $2(1.53 \%)$ & $3(2.3 \%)$ & $5(4 \%)$ \\
\hline \hline Digestive system & $2(1.53 \%)$ & $2(1.53 \%)$ & $4(3 \%)$ \\
\hline \hline Face(ear) & 0 & $2(1.53 \%)$ & $2(1.53 \%)$ \\
\hline \hline Urinary system & $1(0.77 \%)$ & $1(0.77 \%)$ & $2(1.53 \%)$ \\
\hline \hline Others & $3(2.3 \%)$ & $6(4.6 \%)$ & $9(6.9 \%)$ \\
\hline \hline Total & $38(29.24 \%)$ & $92(70.76 \%)$ & $130(100 \%)$ \\
\hline \hline P value & $\begin{array}{c}\chi^{2}=3.132 ; \mathrm{d} . \mathrm{f}=5 ; \mathrm{P}=0.680 \\
\text { By merging small cells as (Clef\&Digest\&Face\&Urin\&Others all as Others) }\end{array}$ \\
\hline \hline
\end{tabular}


High percentage of congenital anomalies was among neonates from consanguineous marriage, (68.46\%), while (31.54\%) among those of non consanguineous one. The most system involved among consanguineous marriage was nervous system $(27.7 \%)$,while $(15.38 \%)$ in non consanguineous ,followed by circulatory system $(18.46 \%)$ in consanguineous and only (3\%) in non consanguineous, followed by chromosomal abnormalities $(4.61 \%)$ in consanguineous and (3\%) in non consanguineous, then genital organs anomalies (3.84\%) in non consanguineous and (1.53\%) in consanguineous. Other systems were involved in small percentages in both groups with the major percentage in consanguineous marriage. These results were statistically significant, $(\mathrm{p}=0.05)$, as shown in Table $(4)$.

Table (4): The role of Consanguinity between father \& mother of neonates with congenital anomalies and system affected

\begin{tabular}{|c|c|c|c|}
\hline System affected & $\begin{array}{l}\text { Consanguinity } \\
\text { No. }(\%)\end{array}$ & $\begin{array}{c}\text { Non-Consanguinity No. } \\
(\%)\end{array}$ & Total No. (\%) \\
\hline Nervous system & $36(27.7 \%)$ & $20(15.38 \%)$ & $56(43 \%)$ \\
\hline Circulatory system & $24(18.46 \%)$ & $4(3 \%)$ & $28(21.7 \%)$ \\
\hline $\begin{array}{c}\text { Chromosomal } \\
\text { abnormalities }\end{array}$ & $6(4.61 \%)$ & $4(3 \%)$ & $10(7.6 \%)$ \\
\hline $\begin{array}{l}\text { Genital organs } \\
\text { anomalies }\end{array}$ & $2(1.53 \%)$ & $5(3.84 \%)$ & $7(5.4 \%)$ \\
\hline $\begin{array}{c}\text { Musculoskeletal } \\
\text { system }\end{array}$ & $6(4.61 \%)$ & $1(0.77 \%)$ & $7(5.4 \%)$ \\
\hline Cleft/lip palate & $4(3 \%)$ & $1(0.77 \%)$ & $5(3.8 \%)$ \\
\hline Digestive system & $3(2.3 \%)$ & $1(0.77 \%)$ & $4(3 \%)$ \\
\hline Face(ear) & $2(1.53 \%)$ & & $2(1.5 \%)$ \\
\hline Urinary system & $2(1.53 \%)$ & & $2(1.5 \%)$ \\
\hline Others & $4(3 \%)$ & $5(3.84 \%)$ & $9(6.9 \%)$ \\
\hline Total & $89(68.46 \%)$ & $41(31.54 \%)$ & $130(100 \%)$ \\
\hline$P$ value & $\begin{array}{l}\chi^{2}=10.77 ; \text { d.f }=5 ; P= \\
\text { By merging small c }\end{array}$ & Digest\&Face\&Uri & rs all as Others) \\
\hline
\end{tabular}

Among 130 mothers studied for malformed neonate, $75(57.7 \%)$ were not used folic acid in the peri conceptional period, while $55(42.3 \%)$ were used it. The most system involved in malformed neonates of mothers not used folic acid was nervous system $(30.76 \%)$ with only $(12.3 \%)$ in those mothers used it, followed by circulatory system (10\%) in mothers not used it, and $(11.53 \%)$ in mothers used it, followed by both 
chromosomal and musculoskeletal system $(3.84 \%)$ in mothers not used it, then anomalies of genital organs (3\%), other systems were involved in small percentages in both groups as shown in Table $(5)$, ( $\mathrm{p}$ $=0.05)$, so this study was statistically significant.

Table (5): Distribution of congenital malformation according to mother`s peri-conceptional folic acid use \& system affected

\begin{tabular}{|c|c||c|c||}
\hline $\begin{array}{c}\text { System } \\
\text { affected }\end{array}$ & $\begin{array}{c}\text { Folic acid use. } \\
\text { No.(\%) }\end{array}$ & No folic acid use. No.(\%) & Total No. (\%) \\
\hline \hline Nervous system & $16(12.3 \%)$ & $40(30.76 \%)$ & $56(43 \%)$ \\
\hline \hline Circulatory system & $15(11.53 \%)$ & $13(10 \%)$ & $28(21.7 \%)$ \\
\hline $\begin{array}{c}\text { Chromosomal } \\
\text { abnormalities }\end{array}$ & $5(3.84 \%)$ & $5(3.84 \%)$ & $10(7.6 \%)$ \\
\hline \hline $\begin{array}{c}\text { Genital organs } \\
\text { anomalies }\end{array}$ & $3(2.3 \%)$ & $4(3 \%)$ & $7(5.4 \%)$ \\
\hline \hline $\begin{array}{c}\text { Musculoskeletal } \\
\text { system }\end{array}$ & $2(1.53 \%)$ & $5(3.84 \%)$ & $7(5.4 \%)$ \\
\hline \hline Cleft/lip palate & $2(1.53 \%)$ & $3(2.3 \%)$ & $5(3.8 \%)$ \\
\hline \hline Digestive system & $3(2.3 \%)$ & $1(0.77 \%)$ & $4(3 \%)$ \\
\hline \hline Face(ear) & $2(1.53 \%)$ & & $2(1.5 \%)$ \\
\hline \hline Urinary system & $2(2.3 \%)$ & & $9(6.9 \%)$ \\
\hline \hline Others & $5(3.84 \%)$ & $4(3 \%)$ & $130(100 \%)$ \\
\hline \hline Total & $55(42.3 \%)$ & $75(57.7 \%)$ & \\
\hline \hline P value & $\begin{array}{l}\chi^{2}=10.67 ; \text { d.f }=5 ; \mathrm{P}=0.05 \\
\text { By merging small cells as (Clef\&Digest\&Face\&Urin\&Others all as Others) }\end{array}$ \\
\hline \hline
\end{tabular}

Among 130 mothers studied for malformed neonate,78(60\%) were delivered by caesarean section, and 52(40\%) were delivered normally. The most system involved in malformed neonates delivered by caesarean section was nervous system $(27.6 \%)$,while $(15.4 \%)$ in those were delivered normally, followed by circulatory system $(12.3 \%)$ in those were delivered by caesarean section and $(9,4 \%)$ in those were delivered normally, followed by chromosomal abnormalities $(4.6 \%)$ in those were delivered by caesarean section and $(3 \%)$ in those were delivered normally, then musculoskeletal system $(3.8 \%)$ in those were delivered by caesarean section and (1.53\%) in those were delivered normally, followed by genital organs anomalies (3\%) in those were delivered normally and $(2.4 \%)$ in those were delivered by caesarean section. Other systems were involved in small percentages in both groups as shown in Table(6), but this study was statistically not significant, ( $p$ valu=0.844). 
Table (6): Distribution of congenital malformations according to mode of delivery of mothers of neonates with congenital anomalies and system affected

\begin{tabular}{|c|c|c|c|}
\hline System affected & $\begin{array}{l}\text { Normal vaginal } \\
\text { delivery. No. }(\%)\end{array}$ & Caesarean section No. $(\%)$ & Total No. (\%) \\
\hline Nervous system & $20(15.4 \%)$ & $36(27.6 \%)$ & $56(43 \%)$ \\
\hline Circulatory system & $12(9.4 \%)$ & $16(12.3 \%)$ & $28(21.7 \%)$ \\
\hline $\begin{array}{l}\text { Chromosomal } \\
\text { abnormalities }\end{array}$ & $4(3 \%)$ & $6(4.6 \%)$ & $10(7.6 \%)$ \\
\hline $\begin{array}{l}\text { Genital organs } \\
\text { anomalies }\end{array}$ & $4(3 \%)$ & $3(2.4 \%)$ & $7(5.4 \%)$ \\
\hline $\begin{array}{l}\text { Musculoskeletal } \\
\text { system }\end{array}$ & $2(1.53 \%)$ & $5(3.8 \%)$ & $7(5.4 \%)$ \\
\hline Cleft/lip palate & $3(2.3 \%)$ & $2(1.53 \%)$ & $5(3.8 \%)$ \\
\hline Digestive system & $1(0.77 \%)$ & $3(2.3 \%)$ & $4(3 \%)$ \\
\hline Face(ear) & & $2(1.53 \%)$ & $2(1.5 \%)$ \\
\hline Urinary system & $2(1.53 \%)$ & & $2(1.5 \%)$ \\
\hline Others & $4(3 \%)$ & $5(3.8 \%)$ & $9(6.9 \%)$ \\
\hline Total & $52(40 \%)$ & $78(60 \%)$ & $130(100 \%)$ \\
\hline$P$ value & \multicolumn{3}{|c|}{$\begin{array}{l}\chi^{2}=2.035 ; \text { d.f }=5 ; \mathrm{P}=0.844 \\
\text { By merging small cells as (Clef\&Digest\&Face\&Urin\&Others all as Others) }\end{array}$} \\
\hline
\end{tabular}

In this study, the major percentage of mothers with malformed neonate had no history of abortion (57.7\%), while (42.3\%) had abortion history. Nervous system was the most system involved in those with a history of abortion (12.3\%),followed by circulatory system (11.53\%) and chromosomal abnormalities (4.6\%) and musculoskeletal anomalies (3\%), then genital organ anomalies $(1.53 \%)$. Other systems were involved in small percentages as shown in Table(7), but this study was statistically not significant, $(\mathrm{p}=0,088)$. 
Table (7): Distribution of congenital malformations according to mother's history of abortion and system affected

\begin{tabular}{|c||c||c||c|}
\hline System affected & $\begin{array}{c}\text { History of abortion } \\
\text { No. }(\%)\end{array}$ & $\begin{array}{c}\text { Non-history of abortion } \\
\text { No. (\%) }\end{array}$ & Total No. (\%) \\
\hline \hline Nervous system & $16(12.3 \%)$ & $40(30.77 \%)$ & $56(43 \%)$ \\
\hline \hline Circulatory system & $15(11.53 \%)$ & $13(10 \%)$ & $28(21.7 \%)$ \\
\hline $\begin{array}{c}\text { Chromosomal } \\
\text { abnormalities }\end{array}$ & $6(4.6 \%)$ & $4(3 \%)$ & $10(7.6 \%)$ \\
\hline \hline $\begin{array}{c}\text { Genital organs } \\
\text { anomalies }\end{array}$ & $2(1.53 \%)$ & $5(3.84 \%)$ & $7(5.4 \%)$ \\
\hline $\begin{array}{c}\text { Musculoskeletal } \\
\text { system }\end{array}$ & $4(3 \%)$ & $3(2.3 \%)$ & $7(5.4 \%)$ \\
\hline \hline Cleft/lip palate & $3(2.3 \%)$ & $2(1.53 \%)$ & $5(3.8 \%)$ \\
\hline \hline Digestive system & $2(1.53 \%)$ & $2(1.53 \%)$ & $4(3 \%)$ \\
\hline \hline Face(ear) & & $2(1.53 \%)$ & $2(1.5 \%)$ \\
\hline \hline Urinary system & \multicolumn{2}{|c|}{$2(1.53 \%)$} & $9(6.9 \%)$ \\
\hline \hline Others & $7(5.38 \%)$ & $2(1.53 \%)$ & $130(100 \%)$ \\
\hline \hline Total & $55(42.3 \%)$ & $75(57.7 \%)$ & \\
\hline \hline P value & $\begin{array}{l}\chi^{2}=9.589 ; \text { d.f }=5 ; \text { P }=0.088 \\
\text { By merging small cells as (Clef\&Digest\&Face\&Urin\&Others all as Others) }\end{array}$ \\
\hline
\end{tabular}

Small percentage of mothers with (3.84\%), and both chromosomal and malformed neonate had history of Diabetes musculoskeletal anomalies (1.53\%). Other Mellitus (15.4\%), (84.6\%) were not diabetic. systems were involved in small percentages The most system involved in neonates with with the majority among those were not diabetic mothers was nervous system diabetic as shown in Table(8), but this study $(5.38 \%)$, followed by circulatory system was not significant, $(\mathrm{p}=0.896)$.

Table (8): Distribution of congenital malformations according to mother's history of diabetes mellitus and system affected

\begin{tabular}{||c||c||c||c||}
\hline System affected & $\begin{array}{c}\text { History of DM } \\
\text { No. (\%) }\end{array}$ & No history of DM No.(\%) & Total No. (\%) \\
\hline \hline Nervous system & $7(5.38 \%)$ & $49(37.7 \%)$ & $56(43 \%)$ \\
\hline \hline Circulatory system & $5(3.84 \%)$ & $23(17.7 \%)$ & $28(21.7 \%)$ \\
\hline \hline $\begin{array}{c}\text { Chromosomal } \\
\text { abnormalities }\end{array}$ & $2(1.53 \%)$ & $8(6.15 \%)$ & $10(7.6 \%)$ \\
\hline \hline $\begin{array}{c}\text { Genital organs } \\
\text { anomalies }\end{array}$ & $1(0.77 \%)$ & $6(4.6 \%)$ & $7(5.4 \%)$ \\
\hline $\begin{array}{c}\text { Musculoskeletal } \\
\text { system }\end{array}$ & $2(1.53 \%)$ & $5(3.84 \%)$ & $7(5.4 \%)$ \\
\hline
\end{tabular}




\begin{tabular}{|c|c|c|c||}
\hline Cleft/lip palate & $2(1.53 \%)$ & $3(2.3 \%)$ & $5(3.8 \%)$ \\
\hline \hline Digestive system & & $4(3 \%)$ & $4(3 \%)$ \\
\hline \hline Face(ear) & $1(0.77 \%)$ & $1(0.77 \%)$ & $2(1.5 \%)$ \\
\hline \hline Urinary system & & $2(1.53 \%)$ & $2(1.5 \%)$ \\
\hline \hline Others & & $9(6.9 \%)$ & $9(6.9 \%)$ \\
\hline \hline Total & $20(15.4 \%)$ & $110(84.6 \%)$ & $130(100 \%)$ \\
\hline \hline P value & $\begin{array}{l}\chi^{2}=1.646 ; \mathrm{d} . \mathrm{f}=5 ; \mathrm{P}=0.896 \\
\text { By merging small cells as (Clef\&Digest\&Face\&Urin\&Others all as Others) }\end{array}$ \\
\hline \hline
\end{tabular}

Among 130 neonates with congenital neonates, followed by chromosomal anomalies, high percentage was recorded abnormalities (4.6\%) among preterm among preterm neonates $(57 \%)$, in relation neonates and (3\%) among full term neonates, with (43\%) among full term neonates. The then anomalies of genital organs (3\%) among most system involved among preterm preterm neonates and (3.2\%) among full term neonates was nervous system (26.9\%), and neonates. Other systems were involved in (16.1\%) among full term neonates, followed small percentages among both groups as by circulatory system (11.53\%) among shown in Table(9), but these results were not preterm neonates and $(10 \%)$ among full term statistically significant as p value $=0.867$.

Table (9): Distribution of congenital malformations according to gestational age of neonates with congenital anomalies and system affected

\begin{tabular}{|c|c|c|c|}
\hline System affected & $\begin{array}{l}\text { Term }>37 \text { wks } \\
\quad \text { No. }(\%)\end{array}$ & $\begin{array}{l}\text { Pre term }<37 \text { wks } \\
\text { No. }(\%)\end{array}$ & Total No. (\%) \\
\hline Nervous system & $21(16.1 \%)$ & $35(26.9 \%)$ & $56(43 \%)$ \\
\hline Circulatory system & $13(10 \%)$ & $15(11.53 \%)$ & $28(21.7 \%)$ \\
\hline $\begin{array}{l}\text { Chromosomal } \\
\text { abnormalities }\end{array}$ & $4(3 \%)$ & $6(4.6 \%)$ & $10(7.6 \%)$ \\
\hline $\begin{array}{c}\text { Genital organs } \\
\text { anomalies }\end{array}$ & $3(2.3 \%)$ & $4(3 \%)$ & $7(5.4 \%)$ \\
\hline $\begin{array}{l}\text { Musculoskeletal } \\
\text { system }\end{array}$ & $4(3 \%)$ & $3(2.3 \%)$ & $7(5.4 \%)$ \\
\hline Cleft/lip palate & $4(3 \%)$ & $1(0.77 \%)$ & $5(3.8 \%)$ \\
\hline Digestive system & $3(2.3 \%)$ & $1(0.77 \%)$ & $4(3 \%)$ \\
\hline Face(ear) & $1(0.77 \%)$ & $1(0.77 \%)$ & $2(1.5 \%)$ \\
\hline Urinary system & $1(0.77 \%)$ & $1(0.77 \%)$ & $2(1.5 \%)$ \\
\hline Others & $2(1.53 \%)$ & $7(5.37 \%)$ & $9(6.9 \%)$ \\
\hline Total & $56(43 \%)$ & $74(57 \%)$ & $130(100 \%)$ \\
\hline $\mathrm{P}$ value & \multicolumn{3}{|c|}{$\begin{array}{l}\chi^{2}=1.872 ; \text { d.f }=5 ; \mathrm{P}=0.867 \\
\text { By merging small cells as (Clef\&Digest\&Face\&Urin\&Others all as Others) }\end{array}$} \\
\hline
\end{tabular}


Among 130 malformed neonates, high percentage was recorded among low birth weight neonates $(53.84 \%)$, followed by normal birth weight neonates (30.76\%), followed by very low birth weight neonates $(9.23 \%)$, then over weight neonates $(6.15 \%)$. Most system involved among low birth weight neonates was nervous system (23\%) and (13.84\%) among normal birth weight neonates followed by circulatory system $(10.76 \%)$ among low birth weight and $(6.15 \%)$ among normal birth weight neonates. Other systems were involved in small percentages with major percentage among low birth weight neonates as shown in Table(10). This study was not significant, $(\mathrm{p}=0.249)$.

Table (10): Distribution of congenital malformations according to birth weight of neonates with congenital anomalies and system affected

\begin{tabular}{|c|c|c|c|c|c|}
\hline System affected & $\begin{array}{l}<1500 \text { gram } \\
\text { No. }(\%) \\
\end{array}$ & $\begin{array}{c}1500-2500 \\
\text { gram No. }(\%)\end{array}$ & $\begin{array}{c}2500-4000 \\
\text { gram No }(\%)\end{array}$ & $\begin{array}{c}4000 \text { gram } \\
\text { No. }(\%) \\
\end{array}$ & Total No. (\%) \\
\hline Nervous system & $5(3.8 \%)$ & $30(23 \%)$ & $18(13.84 \%)$ & $3(2.3 \%)$ & $56(43 \%)$ \\
\hline $\begin{array}{c}\text { Circulatory } \\
\text { system }\end{array}$ & $6(4.6 \%)$ & $14(10.76 \%)$ & $8(6.15 \%)$ & & $28(21.7 \%)$ \\
\hline $\begin{array}{l}\text { Chromosomal } \\
\text { abnormalities }\end{array}$ & & $3(2.3 \%)$ & $7(5.38 \%)$ & & $10(7.6 \%)$ \\
\hline $\begin{array}{c}\text { Genital organs } \\
\text { anomalies }\end{array}$ & & $4(3 \%)$ & $2(1.53 \%)$ & $1(0.77 \%)$ & $7(5.4 \%)$ \\
\hline $\begin{array}{c}\text { Musculoskeleta } \\
1 \text { system } \\
\end{array}$ & $1(0.77 \%)$ & $3(2.3 \%)$ & $2(1.53 \%)$ & $1(0.77 \%)$ & $7(5.4 \%)$ \\
\hline Cleft/lip palate & & $4(3 \%)$ & $1(0.77 \%)$ & & $5(3.8 \%)$ \\
\hline $\begin{array}{c}\text { Digestive } \\
\text { system }\end{array}$ & & $2(1.53 \%)$ & $2(1.53 \%)$ & & $4(3 \%)$ \\
\hline Face(ear) & & $2(1.53 \%)$ & & & $2(1.5 \%)$ \\
\hline Urinary system & & $1(0.77 \%)$ & & $1(0.77 \%)$ & $2(1.5 \%)$ \\
\hline Others & & $7(5.38 \%)$ & & $2(1.53 \%)$ & $9(6.9 \%)$ \\
\hline Total & $12(9.23 \%)$ & $70(53.84 \%)$ & 40(30.76\%) & $8(6.15 \%)$ & $130(100 \%)$ \\
\hline$P$ value & \multicolumn{5}{|c|}{$\begin{array}{l}\chi^{2}=6.636 ; \text { d.f }=5 ; P=0.249 \\
\text { By merging small cells as LBW }(<2500) \times \text { Normal Weight }(=>2500) \text { compared to } \\
\text { (Clef\&Digest\&Face\&Urin\&Others all as Others) }\end{array}$} \\
\hline
\end{tabular}

Blood group(O) was the most common blood group recorded to be associated with congenital anomalies among neonates in Diyala Province $(42.3 \%)$, followed by blood group(B) $(25.4 \%)$, followed by blood group (A) $(19.3 \%)$, then followed by blood group (AB) (13\%). Most system involved among neonates with blood group $(\mathrm{O})$ was nervous system $(17.7 \%)$, and
(10\%) among blood group (B), and (8.5\%) among blood group(A), and (7\%) among blood group (AB), followed by circulatory system $(9.3 \%)$ among blood group (O), and $(5.4 \%)$ among blood group (B), and (4\%) among blood group ( $\mathrm{AB}$ ), and (3\%) among blood group (A), followed by chromosomal abnormalities (3\%) among blood group (O), and (2.3\%) among blood 
group (B), and (1.53\%) among blood group (A), and $(0.77 \%)$ among blood group (AB). Other systems were involved in small percentages with majority among blood group $(\mathrm{O})$ as shown in Table(11),this was descriptive Table.

Table (11): Distribution of congenital malformations according to blood group (ABO) of neonates with congenital anomalies and system affected

\begin{tabular}{|c|c|c|c|c|c|}
\hline System affected & $\begin{array}{c}\mathrm{O} \\
\text { No. }(\%)\end{array}$ & $\begin{array}{c}\text { B } \\
\text { No }(\%)\end{array}$ & $\begin{array}{c}\text { A } \\
\text { No }(\%)\end{array}$ & $\begin{array}{c}\mathrm{AB} \\
\text { No. }(\%)\end{array}$ & $\begin{array}{c}\text { Total No. } \\
(\%)\end{array}$ \\
\hline Nervous system & $23(17.7 \%)$ & $13(10 \%)$ & $11(8.5)$ & $9(7 \%)$ & $56(43 \%)$ \\
\hline $\begin{array}{l}\text { Circulatory } \\
\text { system }\end{array}$ & $12(9.3 \%)$ & $7(5.4 \%)$ & $4(3 \%)$ & $5(4 \%)$ & $28(21.7 \%)$ \\
\hline $\begin{array}{l}\text { Chromosomal } \\
\text { abnormalities }\end{array}$ & $4(3 \%)$ & $3(2.3 \%)$ & $2(1.53 \%)$ & $1(0.77 \%)$ & $10(7.6 \%)$ \\
\hline $\begin{array}{c}\text { Genital organs } \\
\text { anomalies }\end{array}$ & $3(2.3 \%)$ & $2(1.53 \%)$ & $2(1.53 \%)$ & & $7(5.3 \%)$ \\
\hline $\begin{array}{c}\text { Musculoskeletal } \\
\text { system }\end{array}$ & $3(2.3 \%)$ & $2(1.53 \%)$ & $1(0.77 \%)$ & $1(0.77 \%)$ & $7(5.4 \%)$ \\
\hline Cleft/lip palate & $2(1.53 \%)$ & $1(0.77 \%)$ & $2(1.53 \%)$ & & $5(3.8 \%)$ \\
\hline Digestive system & $2(1.53 \%)$ & & $1(0.77 \%)$ & $1(0.77 \%)$ & $4(3 \%)$ \\
\hline Face(ear) & $\overline{~ 1(0.77 \%)}$ & $1(0.77 \%)$ & & & $2(1.53 \%)$ \\
\hline Urinary system & $1(0.77 \%)$ & $1(0.77 \%)$ & & & $2(1.53 \%)$ \\
\hline Others & $4(3 \%)$ & $3(2.3 \%)$ & $2(1.53 \%)$ & & $9(6.9 \%)$ \\
\hline Total & $55(42.3 \%)$ & $33(25.4 \%)$ & $25(19.3 \%)$ & (17(13\%) & 130(100\%) \\
\hline
\end{tabular}

From 130 mothers studied for malformed neonate, 90(69.24\%) were lived in rural areas and $40(30.76 \%)$ who lived in urban areas. The most system involved among neonates in rural areas was nervous system (30.76\%), and $(12.3 \%)$ were in urban areas, followed by circulatory system $(11.54 \%)$ in rural areas and $(10 \%)$ in urban area, followed by chromosomal abnormalities $(5.4 \%)$ in rural areas ,and (2.3\%) in urban areas, followed by genital organs anomalies (3.8\%) in rural areas and $(1.53 \%)$ in urban areas followed by musculoskeletal system $(4.6 \%)$ in rural areas and $(1.53 \%)$ in urban areas. Other systems were involved in small percentages in both groups with majority among rural areas as shown in Table(12) and Figure-2 but this study was not significant as $(\mathrm{p}=0.425)$. 
Table (12): Distribution of congenital malformations according Residency of mothers of neonates with congenital anomalies and system affected affected

\begin{tabular}{|c||c||c||c|}
\hline System affected & $\begin{array}{c}\text { Rural } \\
\text { No.(\%) }\end{array}$ & $\begin{array}{c}\text { Urban } \\
\text { No. }(\%)\end{array}$ & $\begin{array}{c}\text { Total No. } \\
(\%)\end{array}$ \\
\hline \hline Nervous system & $40(30.76 \%)$ & $16(12.3 \%)$ & $56(43 \%)$ \\
\hline Circulatory system & $15(11.54 \%)$ & $13(10 \%)$ & $28(21.6 \%)$ \\
\hline $\begin{array}{c}\text { Chromosomal } \\
\text { abnormalities }\end{array}$ & $7(5.4 \%)$ & $3(2.3 \%)$ & $10(7.6 \%)$ \\
\hline \hline Genital organs anomalies & $5(3.8 \%)$ & $2(1.53 \%)$ & $7(5.4 \%)$ \\
\hline Musculoskeletal system & $6(4.6 \%)$ & $1(1.53 \%)$ & $7(5.4 \%)$ \\
\hline \hline Cleft/lip palate & $3(2.3 \%)$ & $2(1.53 \%)$ & $5(3.8 \%)$ \\
\hline \hline Digestive system & $4(3 \%)$ & & $4(3 \%)$ \\
\hline \hline Face(ear) & $1(0.77 \%)$ & $1(0.77 \%)$ & $2(1.5 \%)$ \\
\hline \hline Urinary system & $1(0.77 \%)$ & $1(0.77 \%)$ & $2(1.5 \%)$ \\
\hline \hline Others & $8(6.15 \%)$ & $1(0.77 \%)$ & $9(6.9 \%)$ \\
\hline \hline Total & $90(69.24 \%)$ & $40(30.76 \%)$ & $130(100 \%)$ \\
\hline \hline P value & $\begin{array}{l}\chi^{2}=4.93 ; \text { d.f }=5 ; \mathrm{P}=0.425 \\
\text { By merging small cells as (Clef\&Digest\&Face\&Urin\&Others all as } \\
\text { Others) }\end{array}$ & \\
\hline \hline
\end{tabular}

\section{Residency of mother.}

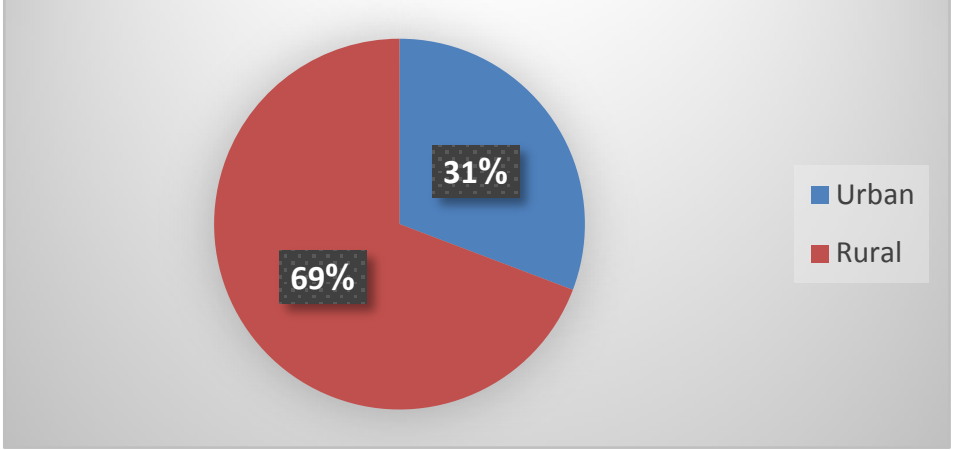

Figure(2): Distribution of congenital malformations according to Residency of mothers of neonates with congenital malformations

From 130 mothers studied for malformed neonates, high percentage $(61.54 \%)$ was recorded among mother`s age 20-35 years, followed by (20.76\%) among mother's age more than or equal to 35 , and then $(17.7 \%)$ among mother's age below 20 years. The most system involved among mother`s age
20-35 years was the nervous system $(26.15 \%)$, and $(9.23 \%)$ among age group more than or equal to 35 , and $(7.7 \%)$ among age group below 20 years, followed by circulatory system $(9.23 \%)$ among age group $>=35$, and (6.15\%) among both ages group20-35 years and $<20$ years, followed by 
chromosomal abnormalities (5.38\%) among age group 20-35 years, and (2.3\%) among age group $>=35$ years. Genital organs anomalies were recorded only among age group 20-35 years $(5.38 \%)$. musculoskeletal anomalies $(3.84 \%)$ were among age group below 20 years, and (1.53\%) among age group 20-35 years. Other systems were involved only among age group 20-35 years as shown in Table(13). This study was not significant, $(\mathrm{p}=0.984)$.

Table (13): Distribution of congenital malformations according to age of mothers of neonates with congenital anomalies and system affected

\begin{tabular}{|c|c|c|c|c|}
\hline "System affected & $\begin{array}{l}\text { Age }<20 \text { years } \\
\text { No. }(\%)\end{array}$ & $\begin{array}{c}20 \_35 \text { Years No. } \\
(\%)\end{array}$ & $\begin{array}{l}\geq 35 \text { years } \\
\text { No. }(\%)\end{array}$ & Total No. (\%) \\
\hline Nervous system & $10(7.7 \%)$ & $34(26.15 \%)$ & $12(9.23 \%)$ & $56(43 \%)$ \\
\hline Circulatory system & $8(6.15 \%)$ & $8(6.15 \%)$ & $12(9.23 \%)$ & $28(21.7 \%)$ \\
\hline $\begin{array}{l}\text { Chromosomal } \\
\text { abnormalities }\end{array}$ & 0 & $7(5.38 \%)$ & $3(2.3 \%)$ & $10(7.6 \%)$ \\
\hline $\begin{array}{c}\text { Genital organs } \\
\text { anomalies }\end{array}$ & 0 & $7(5.38 \%)$ & & $7(5.4 \%)$ \\
\hline $\begin{array}{l}\text { Musculoskeletal } \\
\text { system }\end{array}$ & $5(3.84 \%)$ & $2(1.53 \%)$ & 0 & $7(5.4 \%)$ \\
\hline Cleft/lip palate & 0 & $5(3.84 \%)$ & 0 & $5(3.8 \%)$ \\
\hline Digestive system & 0 & $4(3 \%)$ & 0 & $4(3 \%)$ \\
\hline Face(ear) & 0 & $2(1.53 \%)$ & 0 & $2(1.5 \%)$ \\
\hline Urinary system & 0 & $2(1.53 \%)$ & 0 & $2(1.5 \%)$ \\
\hline Others & & $9(6.9 \%)$ & 0 & $9(6.9 \%)$ \\
\hline Total & $23(17.7 \%)$ & $80(61.54 \%)$ & $27(20.76 \%)$ & $130(100 \%)$ \\
\hline$P$ value & \multicolumn{4}{|c|}{$\begin{array}{l}\chi^{2}=0.033 ; \text { d.f }=2 ; P=0.984 \\
\text { By merging small cells as (Nervous system } X \text { Others All) }\end{array}$} \\
\hline
\end{tabular}

Among 130 neonates with congenital anomalies, most system involved was nervous system (neural tube defect), $32(24.6 \%)$ meningomyelocele, 10(7.7\%) meningocele , and 14(10.7\%) hydrocephalus, followed by circulatory system (congenital heart disease) 28(21.54\%), followed by chromosomal abnormalities (down syndrome) $5(3.8 \%)$, (turner syndrome $3(2.3 \%)$, and (Edward syndrome) 2(1.5\%), followed by musculoskeletal system (limbs anomalies) 7 (5.4\%), followed by genital organs anomalies (hypospadias) 3(2.3\%), (ambiguous genitalia) 3(2.3\%), and (undescended testicle) 1(0.77\%), followed by cleft lip with or without cleft palate $5(3.8 \%)$, 
followed by digestive system(imperforated anus) 2(1.5\%), (anomalies of esophagus) $2(1.5 \%)$, followed by both anomalies of kidney and anomalies of eye, ear, face $2(1.5 \%)$, then followed by other anomalies 9(6.92\%) as shown in Table(14).

Table (14): Distribution of congenital anomalies by system affected according to ICD-10 classification

\begin{tabular}{|c|c|c|}
\hline Types of congenital anomalies & Number & Percentage \\
\hline $\begin{array}{l}\text { Congenital malformation of nervous system (Q00-Q07) } \\
\text { 1- Neural tube defect (Q05) } \\
\text { a- Meningocele } \\
\text { b- Meningomyelocele } \\
\text { 2- }\end{array}$ & $\begin{array}{l}10 \\
32 \\
14\end{array}$ & $\begin{array}{l}7.7 \% \\
24.6 \% \\
10.7 \%\end{array}$ \\
\hline $\begin{array}{l}\text { Congenital malformation of circulatory system(Q20-Q28) } \\
\text { 1- Congenital heart disease }\end{array}$ & 28 & $21.54 \%$ \\
\hline Congenital malformation of eye ,ear , nose (Q10-Q18) & 2 & $1.5 \%$ \\
\hline $\begin{array}{l}\text { Congenital malformation of musculoskeletal system (Q65- } \\
\text { Q79) } \\
\text { 1- Limbs anomalies (Q74) }\end{array}$ & 7 & $5.4 \%$ \\
\hline Cleft lip with or without cleft palate(Q35-Q37) & 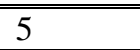 & $3.8 \%$ \\
\hline $\begin{array}{l}\text { Congenital malformation of gastrointestinal system(Q38-Q45) } \\
1-\quad \text { Imperforated anus } \\
2-\quad \text { Congenital malformation of esophagus }\end{array}$ & $\begin{array}{l}2 \\
2 \\
\end{array}$ & $\begin{array}{l}1.5 \% \\
1.5 \% \\
\end{array}$ \\
\hline $\begin{array}{ll}\text { Congenital malformation of genital system (Q50-Q56) } \\
\text { 1- } & \text { Hypospadias( Q54) } \\
\text { 2- } & \text { Undescended testicle (Q55) } \\
\text { 3- } & \text { Ambiguous genitalia( Q56) }\end{array}$ & $\begin{array}{l}3 \\
1 \\
3\end{array}$ & $\begin{array}{l}2.3 \% \\
0.77 \% \\
2.3 \%\end{array}$ \\
\hline $\begin{array}{c}\text { Congenital malformation of urinary system(Q60-Q64) } \\
1-\text { Congenital malformation of kidney(Q63) }\end{array}$ & 2 & $1.5 \%$ \\
\hline $\begin{array}{ll}\text { Chromosomal abnormalities (Q90-Q99) } \\
\text { 1- } & \text { Down syndrome (Q90) } \\
\text { 2- } & \text { Turner syndrome (Q96) } \\
\text { 3- } & \text { Edward syndrome (Q91) }\end{array}$ & $\begin{array}{l}5 \\
3 \\
2\end{array}$ & $\begin{array}{l}3.8 \% \\
2.3 \% \\
1.5 \%\end{array}$ \\
\hline Others (Q80-Q89) & 9 & $6.92 \%$ \\
\hline Total & 130 & $100 \%$ \\
\hline
\end{tabular}

\section{Discussion}

Regarding the gender of neonate with congenital anomalies, males were significantly affected more than females in this study ( $\mathrm{p}$ value $=0.025$ ), and these results were agree with different studies in different countries, including studies done in iran[8], in morocco[9], in Egypt[10], in united kingdom[11], in india[12], and in Alnajaf[13]. So male gender was a risk factor for congenital anomalies, this was probably 
due to genetic and chromosomal abnormalities.

In the present study, regarding address of mothers studied for malformed neonates, high percentage of anomalies were recorded in Baqubah, followed by Alkhalis, Mukdadia, Baladroz, and Khanaqeen. This was the first study done in Diyala Province regarding congenital anomalies, these variations in results between districts were probably due to variations in environmental factors like (pollution, depleted uranium), life style, infection and social factors, also may be due to the site of delivery and drainage. As evident in this study, neonates with multiparas mothers were more affected by congenital anomalies. Most system involved in association with multiparity was nervous system, followed by circulatory system and chromosomal abnormalities, but was statistically not significant, the results were agree with another studies like study done in Romania[14], in Egypt[10], and in Pakistan[15]. These results were probably due to great ratio of high risk pregnancies, difficult labour, and maternal complications among multiparas women.

In the present study and regarding consanguinity, significant percentage of congenital anomalies was recorded among consanguineous marriage $p$ value $=0.05$, The most system affected among consanguineous marriage was nervous system followed by circulatory system and chromosomal abnormalities, the results were agree with another studies studyies done in Iran[16], in India[12] , but disagree with it in regarding the most system affected, in which genital system was the most system involved, This was probably due to genetic and environmental and social variations between countries.

In this study and regarding the use of folic acid during peri conceptional period (prior to and during the first 3 months of pregnancy), we found that there was significant association between the use of folic acid and decrease the risk of congenital anomalies ( $p$ value $=0.05$ ) especially nervous system anomalies (neural tube defects), about $57.7 \%$ of mothers were not used folic acid and 42.3\% were used it, the most system affected with the negative use of folic acid was nervous system, these results were agree with another studies which were done in India[12], in Canada[17], in Egypt[10], and agree with study done in Iran[8], in the effect of nervous system but disagree with that effect on other systems, this was probably due to many environmental and genetic factors and may be due to differences in duration of folic acid use between women.

Regarding the mode of delivery in this study, the most malformed neonates were delivered by caesarean section(60\%), while $(40 \%)$ were delivered normally, the most system involved was nervous system, followed by circulatory system and chromosomal abnormalities. This was not statistically significant, the results were agree with study done in Egypt[10], and disagree with study done in Romania[14]. This was probably due to differences in many factors (age of mothers, life style and complications 
of pregnancy) between countries. History of abortion in the studied group of mothers was recorded in(42\%)of mothers, while $(57.7 \%)$ with no history of abortion, most system affected in relation with history of abortion was nervous system followed by circulatory system and chromosomal abnormalities, this was statistically not significant, the results were agree with another studies done in Romania[14], in India[12], and in Egypt[10]. This was probably due to some severe malformations incompatible with life among mothers with history of abortion.

In this study and regarding mother`s history of diabetes mellitus, (15.4\%) of mothers had history of diabetes, while(84.6\%) did not have it, the most system involved in relation with diabetic history was nervous system, followed by circulatory system and chromosomal abnormalities, this was statistically not significant, the results were agree with another studies done in Romania[14], in Eygpt[10], and in Canada[18], and disagree with studis done in Denmark[19], and in Taiwan[20]. This this was probably due to differences in factors like (age, family history) that predispose mothers to diabetes mellitus by different countries.

In this study, high percentage of birth defects was noted among preterm neonates $(57 \%)$ in comparison to full-term neonates (43\%). The most system affected among preterm neonates was nervous system, followed by circulatory system and chromosomal abnormalities. This was statistically not significant, the results of this study were agree with another study done in Iran[21], in northern Ethiopia[22], and in Nigeria[23].

In this study and regarding birth weights of malformed neonates, high percentage of anomalies were noted among low birth weight neonates(1500-2500 gram)(53.84\%), followed by $(30.76 \%)$ among normal weight neonates(2500-4000 gram), and(9.23\%) among very low birth weight neonates(below1500 gram). The most system affected among low birth weight neonates was nervous system followed by circulatory system and chromosomal abnormalities, This was statistically not significant, results from this study were agree with study done in Nigeria[23], in northern Ethiopia[22], in Egypt[10].

Regarding blood group of neonates with congenital anomalies, high percentage of anomalies $(42.3 \%)$ were recorded among blood group (O), followed by $(25.4 \%)$ among blood group (B), the most system affected among neonates with blood $\operatorname{group}(\mathrm{O})$ was nervous system followed by circulatory system and chromosomal abnormalities, these results were agree with study done in Alnajaf[13].

Regarding residency of mothers of malformed neonates, high percentage of anomalies were recorded in rural $\operatorname{areas}(69.24 \%)$, in relation with $(30.76 \%)$ in urban areas, the most system affected among those neonates in rural areas was nervous system, followed by circulatory system and chromosomal abnormalities. This finding was statistically not significant. These results 
were agree with study done in India[12], this was probably due to many factors: environmental like (pollution, wars), and low socioeconomic status.

In this study, high percentage of anomalies were recorded among mother's age 20-35 years, followed by mother's age more than or equal to 35years, then followed by mother's age below 20 years. The most system affected among age group 20-35 years was nervous system followed by circulatory system and chromosomal abnormalities and genital organs anomalies. Most system involved among age group >=35 years was nervous system, circulatory system and chromosomal abnormalities. This finding was statistically not significant. These results were agree with another study done in India[24], and in Nigeria[23]regarding the nervous system but disagree with circulatory system.

In the present study and regarding the types of congenital anomalies by system affected, the commonest system involved was nervous system, followed by circulatory system, followed by chromosomal abnormalities, genital organs anomalies, musculoskeletal system anomalies, cleft lip with or without cleft palate, digestive system, face(ear ,eye, nose), urinary system anomalies, and then other types of anomalies which not classified. These results were agree with study done in Bahrain[26]. It also agree with study done in india[24] in which nervous system was the most system involved but disagree with the involvement of other systems, The most common anomalies involved the nervous system were neural tube defect (meningomyelocele then meningocele), followed by hydrocephalus, the most common circulatory anomalies were congenital heart diseases, the most common chromosomal abnormalities were down syndrome followed by turner syndrome and then by Edward syndrome, regarding musculoskeletal system anomalies, limbs anomalies were the most common types, the most common anomalies involved the genital organs were hypospadias and ambiguous genitalia, followed by undescended testicle, cleft lips with cleft palates were involved then, followed by digestive system anomalies in which anomalies of esophagus and imperforated anus were the most common anomalies, in urinary system, anomalies of kidney were the most common type, and among anomalies of face, ear anomalies were commonest type, other anomalies which were not classified, were recorded in small percentages, these results were disagree with study done in india by[24], and disagree with another study done in india[12], this probably due to that congenital anomalies differ from one country to another and from one locality to another according to many environmental and genetic factors.

\section{Conclusions}

The study concludes that the most common type of congenital malformations in Diyala province was the nervous system malformations, that congenital malformations were more common in Baquba city and Khalis district, that males were significantly more affected by congenital malformations 
than females, that congenital anomalies were more significant in offspring of consanguineous marriage than of nonconsanguineous one and folic acid usage during peri conceptional period was very important to reduce the incidence of congenital anomalies especially (neural tube defects).

\section{Recommending}

The study recommends increasing public education about the preventable risk factors of congenital malformations through seminars, medical posters and health care organization programs, early prevention, diagnosis and management of common types of congenital malformations by proper antenatal care, advising women in reproductive age group about the benefits of folic acids in preventing neural tube defects, encouraging pre-marital counseling on the subject of parental consanguinity and looking after depleted areas which suffered from pollutions and detritus of wars and provide healthy environment, good housing, pure water and healthy diet.

\section{References}

[1] WHO (2016) : Congenital anomalies.

[2] Powel-Griner E, Woolbright A.Trends in infant deaths from congenital anomalies: results from England and Wales, Scotland, Sweden and United States. Int J Epidemiol; 19(2):391-398.

[3]Corsello G \& Giuffrè M. Congenital malformations , The Journal of MaternalFetal \& Neonatal Medicine 2012; 25:25-29. [4]De Graaf, Johanna PS, Eric AP, Bonsel, Gouke J. Inequalities in perinatal and maternal health". Current Opinion in Obstetrics and Gynecology 2013; 25(2):98108.

[5]Antonín S. Congenital anomalies. 2007; http://www.vrozene-vady.cz.

[6]Kliegman R.M \& Stanton B (2015): nelson textbook of pediatrics 20th edition, 2015: 2, p 906.

[7]Czeizel AE. Birth Defects Are Preventable, International Journal of Medical Sciences 2005; 2(3):91-92.

[8]Hosseini MB , Khamnian Z, Dastgiri S , Raad B. S and Ravanshad. Folic Acid and Birth Defects: A Case Study (Iran), Hindawi Publishing Corporation Journal of Pregnancy 2011; Article ID 370458: 1-3. [9]Elghanmi A, Razine R, Berrada R. Gender Difference in Specific Congenital Anomalies, World Journal of Research and Review 2017; 5(4):106-107.

[10]Shawky RM, Sadik DI. Congenital malformations prevalent among Egyptian children and associated risk factors, The Egyptian Journal of Medical Human Genetics 2011; 12:69-78.

[11] Sokal R , Tata LJ and Fleming KM. Sex prevalence of major congenital anomalies in the United Kingdom: A national populationbased study and international comparison meta-analysis, Birth Defects Res A Clin Mol Teratol 2014; 100(2):79-91.

[12]Chowdhury Fazle Mubarak Bari. Spectrum of Congenital Anomalies among Children Attending the Pediatric Departments of Dhaka Medical College Hospital, IOSR Journal of Dental and Medical Sciences 2014; 13(2):20-46. 
[13]Al-Zubaidi KA, Shemki MA, Yahyab WH. Survey of congenital malformation concerned to multiple factors in Al- Zahraa Hospital childbed and brats in Al-Najaf ALAshraf, Journal Contemp Med Sci 2016; 2(7):83-87.

[14]Căpăţînă D, Camelia CG. Risk Factors Associated With Congenital Anomalies in children, ARS Medica Tomitana 2015; 2(21): 105-111.

[15] Jawad S, Haq I, Cheema MR. Role Of Multiparity In Birth Defect, The Professional Medical Journal 2017; 24(8):1241-1244.

[16] Mosayebi Z, Movahedian AH. Pattern of congenital malformation in consanguineous versus non consanguineous marriage in kashan- Iran, eastern mediterranean health journal 2007; 13(4):868-875.

[17]Wilson RD. Pre-conceptional Vitamin/Folic Acid Supplementation The Use of Folic Acid in Combination With a Multivitamin Supplement for the Prevention of Neural Tube Defects and Other Congenital Anomalies, journal of obstetrics and gynecology of Canada 2007; 29(12):10031013.

[18]Allen VM, Armson BA. Teratogenicity Associated with Pre-Existing and Gestational Diabetes, journal of obstetrics and gynecology of Canada 2007; 29(11):927-934. [19]Mills JL. Malformations in Infants of Diabetic Mothers, Birth Defects Res A Clin Mol Teratol 2010; 88(10):769-778.

[20]Chih-Ping Chen. Congenital malformations associated with maternal diabetic, Taiwanese J Obstet Gynecol 2005; 44(1).
[21]Sarkar S, Patra Ch , Dasgupta MK , Nayek K and Karmakar PR. Prevalence of Congenital Anomalies in Neonates and Associated Risk Factors in a Tertiary Care Hospital in Eastern India , Journal Clinical Neonatol 2013; 2(3):131-134.

[22]Mekonen HK ,Nigatu B, Lamers WH. Birth weight by gestational age and congenital malformations in Northern Ethiopia, BMC Pregnancy Childbirth 2015; 15:76.

[23]Anyanwu LC, Danborno B, Hamman WO. Birth Prevalence of Overt Congenital Anomalies in Kano Metropolis: Overt Congenital Anomalies in the Kano, Universal Journal of Public Health 2015; 3(2):89-96. [24]Pattanaik T, Samal $S$ and Jena $T$. Prevalence and Spectrum of Congenital Malformations in a Tertiary Care Centre, Indian Journal of Neonatal Medicine and Research 2016; 4(3):NO01-NO04.

[25] Abdolahi HM, Kargar Maher $\mathrm{MH}$, Afsharnia $\mathrm{F}$ and Dastgiri S. Prevalence of Congenital Anomalies: A Community-Based Study in the Northwest of Iran, International Scholarly Research Notices 2014; Article ID 920940:5.

[26]Al-Arrayed SS. Epidemiological of congenital abnormalities in Bahrain. Eastern Mediterranean Health J 1995; 2(2):248-252. 